\title{
Article
}

\section{Restraint minimisation in mental health care: legitimate or illegitimate force? An ethnographic study}

Mckeown, Michael, Thomson, Gillian, Downe, Soo, Scholes, A, Edgar, F, Price, O, Baker, J, Greenwood, P, Whittington, R and Duxbury, J

Available at http://clok.uclan.ac.uk/30089/

Mckeown, Michael ORCID: 0000-0003-0235-1923, Thomson, Gillian ORCID: 0000-0003-3392-8182, Downe, Soo ORCID: 0000-0003-2848-2550, Scholes, A, Edgar, F, Price, O, Baker, J, Greenwood, P, Whittington, $R$ et al (2020) Restraint minimisation in mental health care: legitimate or illegitimate force? An ethnographic study. Sociology of Health and IIIness, 42 (3). pp. 449-464. ISSN 0141-9889

It is advisable to refer to the publisher's version if you intend to cite from the work. http://dx.doi.org/10.1111/1467-9566.13015

For more information about UCLan's research in this area go to http://www.uclan.ac.uk/researchgroups/ and search for <name of research Group>.

For information about Research generally at UCLan please go to http://www.uclan.ac.uk/research/

All outputs in CLoK are protected by Intellectual Property Rights law, including Copyright law. Copyright, IPR and Moral Rights for the works on this site are retained by the individual authors and/or other copyright owners. Terms and conditions for use of this material are defined in the policies page. 


\title{
Restraint minimisation in mental health care: legitimate or illegitimate force? An ethnographic study
}

\author{
McKeown, M., Thomson, G., Scholes, A., Edgar, F., Downe, S., Price, O., Baker, J., \\ Greenwood, P., Whittington, R. \& Duxbury, J.
}

Accepted for publication: Sociology of Health and IIIness

\begin{abstract}
Coercive practices, such as physical restraint, are used globally to respond to violent, aggressive and other behaviours displayed by mental health service users ${ }^{1}$. A number of approaches have been designed to aid staff working within services to minimise the use of restraint and other restrictive practices. One such approach, the 'REsTRAIN Yourself' (RYS) initiative, has been evaluated in the UK. Rapid ethnography was used to explore aspects of organisational culture and staff behaviour exhibited by teams of staff working within 14 acute admission mental health wards in the North West region of the English NHS. Findings comprise four core themes of space and place; legitimation; meaningful activity; and, therapeutic engagement that represents characteristics of daily life on the wards before and after implementation of the RYS intervention. Tensions between staff commitments to therapeutic relations and constraining factors were revealed in demarcations of ward space and limitations on availability of meaningful activities. The physical, relational and discursive means by which ward spaces are segregated prompts attention to the observed materialities of routine care. Legitimation was identified as a crucial discursive practice in the context of staff reliance upon coercion. Trauma informed care represents a potentially alternative legitimacy.
\end{abstract}




\section{Introduction}

There is international interest in minimising restrictive practices within mental healthcare (Duxbury 2015). This paper reports on a rapid ethnography situated within a larger evaluation of the ReSTRAIN Yourself (RYS) initiative within mental health inpatient wards (Duxbury et al. 2019a). The ethnographic account provides a contextual overview of the range of environments in included mental health wards; focused attention to specific cultural aspects that could theoretically have been influenced by the RYS approach; and general description of changes occurring on the wards over the time of the study. We explore the legitimacy of restrictive practices in psychiatric services through a sociological lens.

Sociologists have long held an interest in the study of mental illness as a form of social deviance and its relationship to wider society (Rogers and Pilgrim 2014). Classically, Goffman (1961) studied the micro-relational interactions of psychiatric institutions, highlighting how various practices resulted in forms of moral degradation and a mortification of self for detained inmates. Then and now, restrictive practices are deployed within psychiatric services enmeshed with broader systems of governance and control (Foucault 2003, Rose 1996). Notions of epistemic injustice and violence (Fricker 2007) have been taken up in critique of mental health services, whereby psychiatric knowledge stands accused of implicit violence and legitimation of explicitly violent acts such as physical restraint or forced medication (Liegghio 2013, Russo and Beresford 2015). Hence, for Holmes and colleagues (2013: 9) the focus should be on the extent to which violence is 'bred in the practices' of organisations. Furthermore, recent austerity policies have diluted resources, particularly ward staffing levels (Baker et al. 2016), exacerbating restrictive ward cultures (Docherty and Thornicroft 2015). 
Increasingly, inpatient mental health services have become characterised by compulsion and coercion (Szmukler 2015). This has been criticised by progressive organisations, staff and service users (Rose et al. 2015, 2017), prompting initiatives to minimise restrictive practices (Duxbury 2015, Moran et al. 2009, Muir-Cochrane and Gerace 2016, Paterson and Duxbury 2007, Watson et al 2014). Systematic programmes of change including the US Six Core Strategies (6CS) (LeBel et al. 2014), from which RYS is adapted for the UK context, and Safewards (Bowers 2014) have been evaluated internationally (Ashcraft and Anthony 2008, Barton et al 2009, Borckardt et al 2011, Bowers et al. 2015, Lewis et al. 2009, Putkonen et al 2013). These initiatives share a prevention-oriented focus on improving relational care, de-escalating aggression and violence and debriefing teams following use of restraint. RYS specifically emphasises trauma-informed care, acknowledging the harm that restraint can cause. The ethnography aimed to explore ward cultures in relation to experiences of the use of restraint and the impact of the RYS intervention.

\section{Methodology}

This study took place on 14 acute mental health wards within 7 NHS Trusts across the North West of England. Half of the wards, one in each Trust, received the RYS intervention. The impact of this was evaluated using a range of measures within a non-randomised trial. Data collection took place between July 2014-February 2016. A qualitative dimension of the study, comprising rapid ethnography observations and semi-structured interviews with purposive samples of staff and service users, undertaken by researchers attending each ward at two intervals, before and after the intervention phase within each Trust.

Rapid ethnography is an intensive, shortened form of ethnographic data collection designed for researching a slice of organisational life and workplace cultures (Baines and Cunningham 2011, Handwerker, 2001). It has been utilised in health care research where time, 
budgetary, or other pragmatic considerations militate against more extensive anthropological study (Bentley et al. 1988, Johnson and Vindrola-Padros 2017). The approach aims to maximise depth of observation and analysis, even though the period of data collection is relatively short compared with classic ethnographies. Despite various criticisms, robustly conducted rapid ethnographies can usefully inform policy and practice (Vindrola-Padros and Vindrola-Padros 2018).

Two researchers undertook the majority of the ethnography: research assistants from a psychology background (initials) and with lived experience of being detained on mental health wards (initials). Senior researchers (initials) were involved for at least one day of each observation period, and provided regular support and supervision for the research assistants. Having a mixture of service user and professional inputs represented in our team allowed for a richly reflexive relationship to the data (Hammersley \& Atkinson 2007). Prior to the study all members of the research team were committed to the possibilities for restraint reduction and were to a varying degree, critical of coercive practices.

The researchers were present on the wards at each data collection interval for approximately a week, mirroring full staff-shifts and observing key practices such as staff team handover, medication rounds, and lunchtime activity. Ethnographic field notes, structured using Spradley's (1980) framework, were written away from the ward at break-times and end of shift each day. In total, across all the wards and both time intervals, 162 interviews were undertaken; 130 with staff, 32 with service users. The staff sample included different levels of work experience, gender and ages, grades and professional roles. The majority of the staff comprised registered mental health nurses and health care assistants (HCAs), all of whom were involved in restraint practices. Service user participants included a mix of age, gender and diagnosis. Interviews aimed for a conversational, open style, exploring aspects 
of the participants' experiences of the ward environment and restraint practices. They also sought to reveal attitudes towards coercive care, its context and personal impact. In the interviews undertaken after the intervention, experiences of RYS and attempts to change practice were explored. Interviews varied in length from approximately 20 to 90 minutes, were audio-recorded and transcribed in full. Transcripts and emergent analysis were not shared with participants for pragmatic reasons of limited resources.

Field notes relating to 1260 hours of person-observation and interview data were analysed thematically (Braun and Clark 2005). Analysis was undertaken by the four researchers involved in the ethnographic data collection. Initially, two researchers (initials) independently coded the data via iterative reading and re-reading. A coding framework was defined and refined. Codes were then merged and synthesised into key themes that represented the entire data set. Identified themes were agreed with the other two team members involved in data collection (initials), and finally with the whole team. Findings are presented and illustrated with field note excerpts and quotes from interviews. Additional analysis of interview data is reported elsewhere (Duxbury et al. 2019b). Analysis was supported using MAXQDA software (version 11).

As with any ethnography, transferability of findings is limited due to the interpretive emphasis on people and relations within a particular context. The relative brevity of data collection under rapid ethnography has been criticised in some quarters, including the potential to constrain reflexivity, but commentators within the health field believe this need not compromise quality (Vindrola-Padros and Vindrola-Padros 2018). Our study addresses these concerns, with the volume of interview data counterbalancing relative brevity of observations. 
The study was funded by the Health Foundation and received ethical approval via the Health Research Authority (ref. 14/YH/0164).

\section{Results}

The accounts described here represent characteristics of life across the wards, before and after implementation of the RYS intervention. On the intervention wards a number of positive changes were observable. However, the extent of these varied across the different wards and certain negative characteristics of culture and practice persisted beyond the intervention. The findings are presented in four core themes: space and place; legitimation; meaningful activity; and, therapeutic engagement; each with pre and post implementation observations.

\section{Space and Place}

All of the wards differed in design. Tellingly, all were initially informally demarcated into service user and staff space, often comprising quite different worlds. Staff spent most time in office areas with service users congregating in areas proximal to the office. Smaller quiet rooms or TV rooms were under-utilised. Typically, offices had large windows allowing observation of nearby areas, but also constituting a physical barrier between service users and staff. On a few of the wards, a large day room was adjacent or near to the office. Interaction between staff and service users was often constrained by locked office doors, and occupation of space by staff that operated to exclude service users. HCAs were more likely to be visible within main ward spaces but, even when senior and other staff were out of the office, they regularly kept to their own 'world' with minimal relational contact; present but not available: 
The pattern on this ward appears to be that 'regular' staff (especially nurses) are in the office/clinic/reviews (behind closed doors), and agency staff are the only visible members on the ward. Their visibility is purely for instrumental tasks such as facilitating smoking, doing observations, and they rarely engage with service users, unless the service user initiates. Observation: Site 7_intervention_T1

Whilst in living room with service users for a minimum of 1.5 hours we saw a staff member fleetingly twice conducting observations, again not interacting with service users. Observation: Site 6_intervention_T2

Staff use of space was bound up with simultaneous super-valuation of, and irritation with, record-keeping. This and other work pressures were often related in tandem with bemoaning perceived inadequate staffing levels and associated management support. Positive managerial support for their work, and specifically involvement in change practices, was appreciated but not uncommonly ward staff felt that managers were spatially and relationally distant from practice, or even that they were an active impediment to change. Nursing staff claimed that they were under great pressure to complete care planning and other records associated with (they believed) oppressive risk aversion. They often declared that insufficient data entries on the computerised record would be viewed as a disciplinary offence, justifying lengthy periods spent in the office. Alternately, a different sort of defensive practice involved staff protecting themselves from threat or possibilities for emotionally laborious work by taking refuge in their offices (often justified by the need to complete the paperwork they professed to dislike): 
Staff although friendly (mostly) appear to hide in their [offices]. I feel this environment is too comfortable, that staff are somewhat reluctant to leave this area, particularly as the atmosphere on the ward is tense. Observation: Site 6_intervention_T1

The office door was pivotal in the demarcation of ward space, offering a largely impermeable shield from service users. This portal was defended consistently and vigorously, adding to an observed 'us and them' dynamic. At times we observed what we considered to be disrespectful denial of entry or refusal of requests. In the context of a study evaluating approaches to minimise aggression, this appeared provocative and self-defeating:

I witnessed several times, service users being ignored by staff. This is done in a few ways. Service users knock on the office door and get no reply. If a staff then comes out of the office, they make no eye contact with the person and ignore what they're saying. This is rude and horrible to watch. It clearly is a source of great irritation amongst service users and is talked about amongst themselves. Observation: Site 6_intervention_T1

A subsequent, recurring behavioural refrain on more than one ward involved increasingly frustrated individuals resorting to shouting outside or kicking the office door.

After implementation of the intervention, there was little observed change in the overall demarcation of space on most of the wards observed. The two 'worlds' were still clearly evident. In some cases, change may have been difficult due to factors outside of staff control: 
In terms of the occupation of space on the ward, nurses remain behind closed doors either in reviews, in the clinic or behind a computer. They have little to no interaction with service users. However, today I have been made aware that the ward has been placed on a risk register due to the low level of qualified staff (only 3 full-time qualified nurses on the rota). Observation: Site 6_intervention_T2

There were, however, some minor, though perhaps culturally profound changes in some wards, rendering the boundaries between staff and service user space slightly more permeable:

Most staff are still in the office but the door is now open. Observation: Site 4_intervention_T2

Leaving the door open is a powerful symbol of invitation, indicative of an awareness of the importance of design and utilisation of space.

\section{Legitimation: justifying restraint}

It was rare to hear unprompted staff talk about restraint. When it did happen, it was usually observed in office spaces in periods immediately following a restraint episode. Justification was the primary focus of this talk, informed by particular views or judgements on individuals or specific groups of service users. Before implementation, some staff claimed more legitimacy in restraining service users who came from certain populations, such as individuals diagnosed with personality disorder who self-harmed. Furthermore, such 
individuals were frequently typified as manipulative' or 'attention-seeking' (within handovers for instance), especially following restraint incidents. They were not seen as 'deserving', and their behaviour was seen as selfish and disruptive to the smooth running of the ward. Restraint was therefore believed to be the only reasonable option for control and restoration of order:

Conversation with staff engaged in art activity on the ward. A service user who refused to take part was spoken about after she walked away. Staff said this was typical of her because she was a 'self-centred PD patient' who also abused drugs and alcohol and was the sort of person who would inevitably become aggressive if she didn't get what she wanted. And then the use of physical restraint would be equally inevitable. Observation: Site 4_intervention_T1

Other justifications involved protecting staff and other service users from distress at the visible signs of self-harm. On one unit, a young woman was restrained and marched down the corridor to her bedroom:

$X$ explained to me that it is documented in Y's care plan that she is not allowed in the dining room at meal times, and that if she is in the communal areas she is to cover her arms. This is due to her severe self-harm wounds that expose muscle tissue, and that $Y$ regularly opens these old wounds. She commented that some service users (and staff) get very distressed at the sight of her wounds. $X$ explained that $Y$ was on 1-1 observations with a new HCA [Health Care Assistant] to the ward, who wasn't aware $Y$ wasn't allowed to eat in the dining room at meal times, nor enter communal areas without covering her arms. Staff then intervened when $Y$ was in the dining 
room during the meal time, which she reflected led to the incident of restraint. Observation: Site 2_Intervention_T1

This is not to say that all staff viewed all restraint as legitimate at all times. Instead, certain staff had misgivings about the use of restraint, and did not view the experience of restraining another person positively. Some acknowledged the weakness of legitimation appeals that justified restraint usage as a last resort:

... and we always used to use restraint as a last resort but I think we always used to think we did, rather than actually do it. Interview: Site 1_Intervention_Staff 3_T2

Rationalising restraint as a legitimate, last resort intervention both vindicated staff actions, and consoled those who would rather not administer coercive practices. Addressing these concerns was a central part of the RYS intervention. Such justifications were reinforced by some service users, stating that restraint may have been the correct response on occasion to maintain safety and a sense of security:

Conversation with service user following completion of questionnaire exploring views on experiences of different forms of coercion. This person intensely disliked being subject to physical restraint but spoke of circumstances when they felt it was warranted. This would be either when they had personally been aggressive or out of control, or when applied to other service users they were fearful of or who were seen as disruptive for everyone. Observation: Site 3_intervention_T1 
In this sense, service users' views on legitimacy of restraint reflected hindsight awareness about personal behaviour, as well as surfacing concerns about collective social relations. It could equally result from uncritical adoption of staff beliefs about legitimacy. Individuals certainly did not appreciate being subject to coercion or restraint at the time it occurred, particularly if this was deemed oppressive, or undertaken in public view.

The RYS intervention included education about the sort of complex needs represented by a diagnosis of personality disorder. Interestingly, many staff engaged positively in this, keen to develop new knowledge and skills within an external learning environment. Indeed, staff were observed to behave and communicate differently away from ward settings, being much more likely to offer positive, empathic reflections upon service users under their care. This included compassionate insights into the possible traumatic antecedents of self-harm or aggression and violence, and reflective intimations that management of aggression could have been done differently on occasion. Post-implementation, there were some shifts in attitude about self-harm and restraint across the wards, reflected in both talk and action. Post implementation, staff communication appeared to be less derogatory, and a 'hands off' approach was observed more often:

Staff have tried to de-escalate (to no effect), and tried to spend time de-escalating in the courtyard (again to no-effect). Some conversation in the office occurred around potentially administering intra-muscular medication, and the doctor has written this up. However, this wasn't acted on. The person was allowed to vent, and be quite severely hostile to staff without being reprimanded or challenged. The Ward Manager has reflected learning 'when not to do anything' has been a positive aspect of the project. Observation: Site 1_intervention_T2 
Staff also began to implement more formal aspects of the RYS approach including ward community meetings designed to attend to service users' concerns, and imaginative approaches to address and record aspects of ward atmosphere and safety. In conversation, many staff appreciated the trauma-informed aspects of the project and better understanding of reasons why people may harm themselves:

I think there's less anxiety around patients being admitted that self-harm. I think it's given people a different way of thinking about it. Interview: Site 4_Intervention_Staff 1_T2

For some, this had led to 'epiphanies' about their own practice. Concomitantly, staff grew in confidence to challenge decisions made about care and attempt to prevent further psychological and physical harm, although potential for positive risk-taking was not always accepted within teams and on occasion was countermanded by psychiatrists.

\section{Meaningful Activity}

One of the ways in which relatively settled and unsettled wards could be distinguished was the availability of organised and meaningful activities. Better quality activities were observed if Occupational Therapy (OT), psychology, or Activities Co-ordinators were attached to the ward teams. Staff associated with the provision of meaningful activities were notable for their motivation. They experienced job fulfilment and exuded enthusiasm in ways mainstream staff did not: 
The activities coordinator spoke with us for quite a while about the various activities and complexities of involving as many service users as possible in activities. Her energy is contagious, and she is clearly passionate about the welfare of service users. Observation: Site 4_intervention_T1

Activities included both 'therapies', such as Cognitive Behavioural Therapy (CBT) and opportunities for creativity or practical occupation, such as gardening. Facilitating staff made explicit connections between activities and a calming effect on ward atmosphere:

\footnotetext{
$X$ also commented that he likes activities that are multisensory, as he feels these are more therapeutic. He reflected that something as simple as mask-making can be particularly therapeutic ... the mod-rock and clay is very messy and tactile [the activity] distracts service users for a few moments from their complex issues. Observation: Site 2_intervention_T1
}

Some units provided specialist activities with invited guests such as professional musicians:

The two musicians invited questions from service users, called everyone by name, and made an effort to engage with every person who attended the activity. For the first time in many days, the focus of conversation was not on emotions, feelings, mental health status, medication etc. but about a topic completely interesting to everyone in the room. Observation: Site 3_intervention_T2

In contrast, there were substantial gaps on many of the wards: 
This is the 7th ward I have visited with this project, and this is the first time I have met a full-time Clinical Psychologist on the ward, who runs therapy related sessions $x 3$ a week. This is the first time I have observed and seen available CBT principles. Only one or 2 other wards in other Trust's, mention mindfulness briefly as a way to manage psychological distress. Observation: Site 4_intervention_T1

Ward resources for activities were fairly limited. Intersecting with concerns regarding use of space, a lack of things to do led to people isolating themselves in bedrooms with iPads or mobile phones. Activity rooms were often locked, restricting access to activities at service users' own behest. Moreover, activity rooms were often poorly stocked. We were regularly told that service users produced artwork for the walls, but such work was rarely displayed. Accessible space outside the wards was usually an unkempt area, often used for smoking. One unit had no outside space whatsoever. On another, the staff were fearful of occupying the garden space because the trees were identified as ligature points - confirming tendencies for risk aversion.

Some advertised schedules of activities were subject to substantial cancellations or postponements because of staffing or other resource pressures. Not all service users appreciated what was on offer, felt patronised, or covered up their abilities in order to avoid engaging in activities they considered meaningless. Boredom was reported frequently.

Despite being highly relevant to the RYS intervention, the quality and extent of meaningful activities on the implementation wards did not always improve. If anything, on some of the wards, activity levels reduced due to key staff absences. We observed very few attempts 
made by staff who were not activity specialists to facilitate the activity timetables.

Conversely, where activities were better supported, there were attempts to play to staff interests and talents:

$X$ 's [staff member] been assigned to do the gardening, and the garden's great. [...] $Y$ [staff member] he's really into artwork, he's got an Art Degree so he's been doing [art classes]. Interview: Site 4_Intervention_Staff 8_T2

On the two wards with high observed levels of positive activity pre and post intervention, this seemed almost entirely due to particular staff, rather than a team commitment. This suggests that responsibility for meaningful activity vested in specific individuals or departments may paradoxically lead to reductions in activities if nursing staff do not see this as their professional concern.

\section{Therapeutic Engagement}

Even before the implementation of the RYS intervention there was evidence of particular aspects of ward culture reinforcing therapeutic relations between staff and service users. The quality of therapeutic relations was most obvious when staff spent more time engaged in face to face contact with service users. This theme of therapeutic engagement therefore interacts with our observations of use of ward space and opportunities for meaningful activity.

Staff who spent most time with service users tended to be the least qualified team members, such as HCAs and, in one Trust, apprentices. Conversations started usually with banter, 
discussing TV or non-clinical matters, and service users valued this 'ordinary' talk. Positive social and therapeutic relations were seen to be undermined by resource issues affecting staffing levels, including difficulties associated with deployment of temporary staff. Service users on most wards pre-intervention regularly bemoaned lack of time with staff, or that interactions were clinical and impersonal. Many felt staff did not know them personally, or did not attempt to get to know them, leading to frustrations, and an 'us and them' mentality:

\begin{abstract}
$X$ commented that there was a severe lack of contact time between staff and service users. He felt that before asking clinical questions (e.g. do you think the television is talking to you as a first line of questioning) staff should get to know the person. Observation: Site 2_intervention_T1
\end{abstract}

The traumatic impact of restraint had individual and collective impacts upon therapeutic relations and ward atmosphere. There were numerous examples of people being distressed by being restrained or subject to coercion, with staff also upset by these incidents. Feelings of powerlessness or not being listened to often preceded expressions of aggression and violence and were then compounded by the experience of being restrained and forcibly medicated. Traumatic impact also extended to witnesses:

$X$ reported that she had seen the individual 'dragged, kicking and screaming' to the clinic and that it was very traumatic to her. She stated that every time she hears the alarm now, she gets a 'funny feeling' in her chest. Observation: Site 3_intervention_T1 
The application of staff-initiated rules, such as frequency of access to smoking space or egress from ward, often precipitated conflict and aggressive responses. On many wards the flexibility or rigidity with which rules were applied could depend upon which staff were on duty or who was in charge. Service user frustrations were compounded if the notional arbiter of the rule was typically not present to enforce or explain it.

Post intervention, problems with understaffing and with the weight of administrative work persisted on most wards. Congruent with core relational aspects of RYS, there was, however, evidence of improvement in therapeutic engagement. On some wards staff were spending substantially more time with service users. However, it was still mostly HCA's and Activity Co-ordinators who provided most direct contact:

HCAs on this ward are an obvious invaluable asset to the ward, and have fantastic rapport with service users. They can always be seen in service user areas, and are very diligent on their 1-to-1 observations, in terms of not restricting the movement of service users to suit themselves (which I have observed on other wards), and despite being on 1-to-1 observations for a prolonged period of time (an entire shift), they appear alert. HCAs can be seen actioning requests, nipping in the bud people knocking on the office door for things such as: chargers, money, access to their bedroom. This is something that is definitely different [from before implementation]. Observation: Site 3_intervention_T2

\section{Discussion}

This study took place in a context of constraining structural and resource factors, including managerial reorganisations and service relocations for some. However, in all the study 
wards, both before and after the RYS implementation, culture and practices shared a number of characteristics. We focus here on the staff legitimation practices, first locating these within consideration of the spaces of psychiatric care within which the study took place and the mundane materialities of these ward settings, the objects and built environment, and the ordinary, everyday interactions therein.

\section{Psychiatric inpatient spaces}

The findings indicate constraints on time staff spend in direct encounters with service users, limiting supportive or therapeutic relationships, confirming previous research into mental health wards (Gilburt et al 2008, Quirk and Lelliott 2001). The noted temporal rhythms and spatial demarcations of ward life connect with key dimensions of Spradley's (1980) framework and previous commentary on space and place in relation to mental health in society and psychiatric services (Parr 2011, Wolch and Philo 2000), and inpatient services particularly (Curtis et al. 2007, Spandler 2009).

Within a general delineation of health landscapes, Curtis (2004) distinguishes between therapeutic 'landscapes' (Gesler and Kearns 2002) and landscapes of power. The former are informed by understandings of sense of place or identity, and emotional response to settings, while the latter are concerned with socio-political control within socially defined spaces. Both analyses of space are relevant to our observations of the ward spaces in our study, with a demarcation of staff and service user space on all of the wards. This indicates tensions between therapeutic and coercive potentialities.

The typical staff space was ward offices that service users seldom entered and other areas mainly occupied by service users that staff inhabited infrequently. These two-worlds recall 
Goffman's (1961) classic observations of institutional care, where distinctions between staff and patient identity were realised through symbolic and ritual interactions and maintained within the spatial and cultural demarcations of asylum life. The offices in our study were sites of intensive commentary on behaviour and relations between care team and service users, collated within computerised care records. Commentators have ascertained a 'fictional' dimension to such narration activities, given that they involve writing about people or making assessments without them, and recording aspects of ward life without spending substantial time present to witness actual behaviour (Coffey et al. 2017). In this sense, whole systems of bureaucracy act to limit actual engagement, with attention to the perceived importance of 'paperwork' justifying time spent away from service users.

Staff reported oppressive institutional expectations of servicing the record keeping process (see also Brooks et al. 2018), believing (with some justification) that not to make sufficient data entries would attract managerial disapproval. It was seldom felt that lack of real interpersonal engagement would be similarly subject to supervisory discipline. Weber's (1905/1958) iron cage of bureaucracy offers an interesting means for making sense of the observation that bureaucratised care was a seductive diversion from more relational alternatives. For Weber such developments reflect a more general increasing rationalisation of social life under western capitalism. In the contemporary ward systems, this tendency towards rationalisation is framed by governance systems of risk management within a wider frame of risk society (Beck 1992, Lupton 1993); wherein the desire or necessity to be spared from risk has become a defining feature of modernity, and the mental health system is prominent within this.

In this context, there is also a prevailing intolerance of uncertainty despite the actual existence of maximal uncertainty (Bauman 2000). Drawing upon Bauman's work, critical 
healthcare commentators have noted the extent to which the organisation and resourcing of modern services has resulted in a destabilised workforce, with colleagues and services users never sure of who they will be working with from one day to the next (Randall \& McKeown 2014). Bureaucratic systems offer a phantasy of certainty, and failings are catastrophized rather than viewed as a fact of life. This state of affairs stokes staff anxieties, predicating and justifying a perceived and actual blame culture, further perpetuating staff's commitment to servicing the bureaucracy and legitimising such efforts with the concomitant retreat into particular spaces.

\section{Everyday materialities}

Early theorising of a sociology of space notes that various notable sociologists, such as Goffman, have worked with a saliency of space, spatial arrangements, and the interactions therein, ordered by hospital architecture, for making sense of human behaviour and resource mobilisation (Prior 1988). The physical, relational and discursive means by which ward spaces are segregated prompts attention to the observed materialities of routine care. Recent writing on the materialities of care has considered a number of different healthcare settings, highlighting the importance of the seemingly mundane, such as objects and taken for granted elements of the built environment, for making sense of the practices, relationships, spatiality and cultures of care (Buse et al. 2018, Maller 2015, Nettleton et al. 2018). Brownlie and Spandler (2018: 267) have extended this concern with the mundane materialities of care to the informal mental health context, and their conclusions regarding the value of shared space appear to also be relevant to professionalised spaces, with 'the possibility for small acts of care to emerge in and through sharing the most ordinary of spaces and things'. Seen from this perspective, initiatives such as RYS have been conceived in reaction to aspects of discipline, control and bodily regulation that have been quite literally built in to the psychiatric hospital space of the ward settings studied here (Prior 
1988). In this sense, RYS represents a counter-discourse to the prevailing discursive practices that legitimate use of physical restraint.

Thus, our observations of the changes in practice resulting in the seemingly small, inconsequential act of leaving of the office door open, and how this allowed for less demarcated ward space and freer contact between staff and service users, represents just such a scenario where a simple material change can have a profound impact. More broadly speaking, the observed commonplace demarcation of ward space is enabled by building design features, offices, day areas and corridors that, whilst representing modern incarnations, resonate with older spatial configurations harking back to asylum times. Similarly, the social relations of inpatient ward care also continue to have characteristics of the rituals of mortification of self that Goffman observed in those latter-day institutions.

\section{Exculpatory legitimation}

Ours and other studies show that staff in mental health services collectively and individually engage in legitimation narratives regarding restrictive practices, in particular, and psychiatry more generally (Gadsby 2018, Perkins et al. 2012). Burstow (2016) offers a scathing critique of the wider influence of legitimation narratives within the mental health system as part of a broader re-emergence of anti-psychiatry scholarship and critical mad studies (Le Francois et al. 2013). These justifications for how things are done are often linked to 'otherness' archetypes; though the 'othered' are not a homogenous group (Parr 2000). In this way, selfother distinctions and the stories staff tell bolster a sense of having acted correctly, and minimise dissonance between personal ideals, professional practice and associated negative emotions; such as revulsion at the violent or oppressive aspects of restraint. Similarly, these narratives operate to induct and socialise staff into prevailing cultures of coercion and control (Chapman 2014, McKeown et al. 2019). 
Generally speaking, violence is viewed as legitimate if principles of fairness and procedural justice prevail, and this sustains cooperation with, and deference to, the exercise of power (Tyler 2006). In the mental health context, when belief in the just administration of power diminishes, trust is highly likely to break down between individuals or communities and services and the practitioners within them. This, in and of itself, may explain crises of legitimacy in a context of service-sanctioned restrictive practices, and, perhaps, escalations of violence in ward settings. Debriefing after violent incidents is a key aspect of RYS, aimed at learning from events. However, debriefing risks defaulting to the telling and retelling of legitimation narratives, becoming more exculpatory than explanatory (Chapman 2014).

Recognition of trauma: an alternate legitimation narrative?

Recognising and responding effectively to trauma is central to RYS and congruent with a contemporary movement towards trauma informed care (Sweeney et al. 2018). Arguably, a turn to more compassionate, relational care should have profound implications for staff and service users alike. This was powerfully noted in Johnston's (2014: 170) auto-ethnographic account of an epiphany in realising the violent trauma of restraint:

It was only when I witnessed the tears and felt emotion pouring down the man's cheek that I realized the performative act and fear behind all of our gestures, commands, and established authority.

A triumph of empathy over otherness indicates an enduring attachment amongst the nursing teams (inclusive of registered nurses and health care assistants) for relational approaches to 
care over the instrumental value of coercion. Improved understandings of self-harming behaviour, for example, open up possibilities to ascribe newly legitimating social meanings to such acts, involving coping with or soothing trauma, rather than invoking revulsion or demonization (Adler and Adler 2011).

The pull to coercion, however, is powerful and subject to social forces arguably beyond the influence of individual nurses and teams. Different use of ward space ought to have been within the remit of RYS to influence, but this appears to have been relatively impervious to change. The staff had to gain faith in the instrumental value of alternative approaches towards volatile and disturbed individuals. In effect, they had to believe that acting differently would actually work in managing or calming the situation. Quite often, they also had to be persuaded that doing different things as a team would be managerially supported and, indeed, legitimated.

\section{Conclusions}

RYS had mixed success in impact upon observed culture and practices at ward level. This is unsurprising given some of the powerful and historically contingent social forces that frame psychiatric services. Our findings support the view that a pervasive psychiatric knowledge system underpins the controlling and coercive nature of everyday practices within services (Foucault 2003) that can be both implicitly and explicitly violent (Liegghio 2013). Social acceptance of the obviously oxymoronic notion of 'coercive care' reflects the fact that legal systems and public opinion approve and legitimate force in psychiatric settings in certain circumstances. Procedural justice concerns regarding proportionality and fairness drive some of this legitimation. However, other factors also play into the legitimated use of violence against service users. Not least of these may be a wider societal concern with risk and danger, exacerbated by austerity driven cuts to staffing and other resources that might 
have enabled alternate, more permissive, less restrictive environments (McKeown et al. 2019). These factors clash with policy and professional rhetoric favouring therapeutic alliance, co-production and democratised relations of care, along with programmes of change management seeking to reduce restrictive practices, such as RYS.

The pressures upon staff teams were substantial, and there is evidence of an interrelationship between diminished resources and deployment of coercive practices (Quirk and Lelliott 2001). Empathic engagement made a difference, particularly in spaces where particular groups of people are 'othered', but various factors transpired to privilege bureaucratic, non person-centred use of staff time. That staff working within such constraints are able to make some positive change is commendable.

Ideally, reducing use of physical restraint and other coercive practices requires epistemic and resource shifts, potentially requiring a rejection of the legitimacy of use of force against psychiatrised individuals. This needs less emphasis upon perceived dangerousness, and a move towards recognition of vulnerabilities and trauma as triggers for mental distress, as well as a shared recognition of humanity rather than otherness. Implicit anxieties about the assumed unpredictability and lack of control of those with mental health problems can help to explain slow progress towards lessening coercive practices. Influential factors are at once mundane, structural, discursive and inter-personal, and thus pose a substantial challenge to progressive change.

1. The terminology of service user has largely supplanted 'patient' in contemporary mental health policy. This is to some extent a reaction to perceived notions of paternalism and biomedical power (Cutcliffe and Happell 2009) within a wider crisis 
of legitimacy, urging a democratisation of care (Prior 2003). An additional concern for critics of psychiatry is the association of such paternalism with medical hegemony and notions of epistemic injustice and violence (Fricker 2007, Liegghio 2013).

\section{Acknowledgements}

Funding: This work was supported by the Health Foundation as part of the Closing the Gap funding stream 2014-2016 (ref 7623).

We are very grateful to all participating staff, service users and NHS organisations and for the helpful comments of two anonymous reviewers.

\section{Contributors}

All authors made substantial contributions to: (1) the conception and design of the study; (2) the collection, analysis and interpretation of data; (3) drafting the article and (4) revising the article. All authors have given final approval of the version submitted for publication. 


\section{References}

Adler, P.A. and Adler, P. (2011) The Tender Cut: Inside the Hidden World of Self-Injury. New York: NYU Press.

Ashcraft, L. and Anthony, W. (2008) Eliminating seclusion and restraint in recovery-oriented crisis services. Psychiatric Services, 59: 1198-1202

Baines, D. and Cunningham, I. (2011) Using comparative perspective rapid ethnography in international case studies: strengths and challenges. Qualitative Social Work, 12, 1, 73-88.

Baker, J., Pryjmachuk, S. and Mental Health Nursing Academics UK. (2016) Will safe staffing in Mental Health Nursing become a reality? Journal of Psychiatric and Mental Health Nursing, 23(2), 75-76.

Barton, S. A., Johnson, M. R. and Price, L. V. (2009) Achieving restraint-free on an inpatient behavioral health unit. Journal of Psychosocial Nursing and Mental Health Services, 47:3440.

Bauman, Z. (2000) Liquid modernity. Cambridge: Polity Press.

Bentley, M.E., Pelto, G.H., Straus, W.L., Schumann, D.A., Adegbola, C., de la Pena, E., Oni, G.A., Brown, K.H. and Huffman, S.L. (1988) Rapid ethnographic assessment: applications in a diarrhea management program. Social Science \& Medicine, 27(1), 107-116.

Borckardt, J.J., Madan, A., Grubaugh, A.L., Danielson, C.K., Pelic, C.G., Hardesty, S.J., Hanson, R., Herbert, J., Cooney, H., Benson, A. and Frueh, B.C. (2011) Systematic investigation of initiatives to reduce seclusion and restraint in a state psychiatric hospital. Psychiatric Services, 62(5), 477-483.

Bowers, L. (2014) Safewards: a new model of conflict and containment on psychiatric wards. Journal of Psychiatric and Mental Health Nursing, 21(6), 499-508. 
Bowers, L., James, K., Quirk, A., Simpson, A., Stewart, D. and Hodsoll, J. (2015) Reducing conflict and containment rates on acute psychiatric wards: The Safewards cluster randomised controlled trial. International Journal of Nursing Studies, 52(9), 1412-1422.

Braun, V. and Clarke, V. (2006) Using thematic analysis in psychology. Qualitative Research in Psychology, 3(2), 77-101.

Brooks, H.L., Lovell, K., Bee, P., Sanders, C. and Rogers, A. (2018) Is it time to abandon care planning in mental health services? A qualitative study exploring the views of professionals, service users and carers. Health Expectations, 21(3), 597-605.

Brownlie, J. and Spandler, H. (2018) Materialities of mundane care and the art of holding one's own. Sociology of health \& illness, 40(2), 256-269.

Burstow, B. (2016) Legitimating Damage and Control: The Ethicality of Electroshock Research. Intersectionalities: A Global Journal of Social Work Analysis, Research, Polity, and Practice, Special Issue: The Ethics and Politics of Knowledge Production, 5, 1, 94-109.

Buse, C., Martin, D. and Nettleton, S. (2018) Conceptualising 'materialities of care': making visible mundane material culture in health and social care contexts. Sociology of Health \& Illness, 40(2), 243-255.

Chapman, C. (2010) Becoming perpetrator: How I came to accept restraining and confining disabled Aboriginal children. In B. Burstow, B. A. LeFrancois and S. L. Diamond (eds) Psychiatry disrupted: theorizing resistance and crafting the (r)evolution. Montreal, QC: McGill/Queen's University Press. pp. 16-33.

Cheetham, J., Holttum, S., Springham, N. and Butt, K. (2017) 'I can see it and I can feel it, but I can't put my finger on it': A Foucauldian discourse analysis of experiences of relating on psychiatric inpatient units. Psychology and Psychotherapy: Theory, Research and Practice, DOI:10.1111/papt.12162 
Coffey, M., Cohen, R., Faulkner, A., Hannigan, B., Simpson, A. and Barlow, S. (2017) Ordinary risks and accepted fictions: how contrasting and competing priorities work in risk assessment and mental health care planning. Health Expectations, 20(3), 471-483.

Curtis, S. (2004) Health and inequality: geographical perspectives. London: Sage.

Curtis, S., Gesler, W., Fabian, K., Francis, S. and Priebe, S. (2007) Therapeutic landscapes in hospital design: a qualitative assessment by staff and service users of the design of a new mental health inpatient unit. Environment and Planning C: Government and Policy, 25(4), 591-610.

Cutcliffe, J. and Happell, B. (2009) Psychiatry, mental health nurses, and invisible power: Exploring a perturbed relationship within contemporary mental health care. International Journal of Mental Health Nursing, 18(2), 116-125.

Docherty, M. and Thornicroft, G. (2015) Specialist mental health services in England in 2014: overview of funding, access and levels of care. International Journal of Mental Health Systems, 9(1), p.34.

Duxbury, J.A. (2015) The Eileen Skellern Lecture 2014: physical restraint: in defence of the indefensible? Journal of Psychiatric and Mental Health Nursing, 22(2), 92-101.

Duxbury, J., Baker, J., Downe, S., Jones, F., Greenwood, P., Thygesen, H., McKeown, M., Price, O., Scholes, A., Thomson, G., Whittington, R. (2019a) Minimising the use of physical restraint in acute mental health services: The outcome of a restraint reduction programme ('REsTRAIN YOURSELF'). International Journal of Nursing Studies, 95, 40-48.

Duxbury, J., Thomson, G., Scholes, A., Edgar, F., Baker, J., Downe, S., Price, O., Greenwood, P., Whittington, R. \& McKeown, M. (2019b) Staff experiences and understandings of the RESTRAIN Yourself initiative to minimise the use of physical restraint on mental health wards. International Journal of Mental Health Nursing, 28, 845-856. 
Foucault, M. (2003) Madness and civilization. London: Routledge.

Fricker, M. (2007) Epistemic Injustice: Power and the Ethics of Knowing. Oxford: Oxford University Press.

Gadsby, J. (2018) Nursing violence, nursing violence. In P. Bull, J. Gadsby \& S. Williams (eds) Critical mental health nursing: observations from the inside. Ross-on-Wye: PCCS Books. pp. 13-29.

Gesler, W. M. and Kearns, R. A. (2002) Culture/Place/Health. London: Routledge.

Gilburt, H., Rose, D. and Slade, M. (2008) The importance of relationships in mental health care: A qualitative study of service users' experiences of psychiatric hospital admission in the UK. BMC Health Services Research, 8(1), 92.

Goffman, E. (1961) Asylums: Essays on the Social Situation of Mental Patients and Other Inmates. New York: Anchor Books.

Hall, J.E. (2004) Restriction and control: the perceptions of mental health nurses in a UK acute inpatient setting. Issues in Mental Health Nursing, 25(5), 539-552.

Hammersley, M. and Atkinson, P. (2007) Ethnography: principles in practice. 3rd ed. London; New York: Routledge.

Handwerker, W. (2001) Quick Ethnography. Walnut Creek, CA: Altamira Press.

Holmes, D., Rudge, T., Perron, A. and St. Pierre, I. (2013) Introduction: (Re)thinking violence in health care settings. In A. Perron, T. Rudge, and D. Holmes (eds.) (Re) Thinking Violence in Health Care Settings: A Critical Approach. Farnham, Ashgate Publishing.

Johnson, G.A. and Vindrola-Padros, C. (2017) Rapid qualitative research methods during complex health emergencies: a systematic review of the literature. Social Science \& Medicine, 189, 63-75. 
Johnston, M.S. (2014) "Telling masculine tales": tracing my embodied experience as a psychiatric ward security guard through ethnographic narrative writing. Sociology Mind, 4(2), 161.

Le Francois, B., Menzies, R. and Reaume, G. (eds) Mad matters: a critical reader in Canadian mad studies. Toronto: Canadian Scholars Press.

Liegghio, M. (2013) A Denial of Being: Psychiatrization as Epistemic Violence. In B. Lefrançois, R. Menzies, and G. Reaume (eds) Mad Matters: A Critical Reader in Canadian Mad Studies. Toronto: Canadian Scholars Press.

LeBel, J.L., Duxbury, J.A., Putkonen, A., Sprague, T., Rae, C. and Sharpe, J. (2014) Multinational experiences in reducing and preventing the use of restraint and seclusion. Journal of Psychosocial Nursing and Mental Health Services, 52(11), 22-29.

Lewis, M., Taylor, K. and Parks, J. (2009) Crisis prevention management: a program to reduce the use of seclusion and restraint in an inpatient mental health setting. Issues in Mental Health Nursing, 30:159-164.

Maller, C.J. (2015) Understanding health through social practices: performance and materiality in everyday life, Sociology of Health \& IIIness. 37, 1, 52-66.

McKeown, M., Scholes, A., Jones, F. and Aindow, W. (2019) Coercive practices in mental health services: stories of recalcitrance, resistance and legitimation. in A. Daley, L. Costa and P. Beresford (Eds) Madness, violence and power. Toronto: University of Toronto Press.

McKeown, M., Thomson, G., Scholes, A., Jones, F., Baker, J., Downe, S., Price, O., Greenwood, P., Whittington, R. \& Duxbury, J. (2019) 'Catching your tail and firefighting': the impact of staffing levels on restraint minimisation efforts. Journal of Psychiatric and Mental Health Nursing, 26, 131-141. 
Moran, A., Cocoman, A., Scott, P.A., Matthews, A., Staniuliene, V. and Valimaki, M. (2009) Restraint and seclusion: a distressing treatment option? Journal of Psychiatric and Mental Health Nursing, 16(7), 599-605.

Muir-Cochrane, E. and Gerace, A. (2016) Containment practices in psychiatric care. In D Holmes, J Jacob and A. Perron (eds) Power and the Psychiatric Apparatus: Repression, Transformation and Assistance (pp. 91-115). New York: Routledge.

Nettleton, S., Buse, C. and Martin, D. (2018) 'Essentially it's just a lot of bedrooms': architectural design, prescribed personalisation and the construction of care homes for later life. Sociology of Health \& Illness, 40(7), 1156-1171.

Paterson, B. and Duxbury, J. (2007) Restraint and the question of validity. Nursing Ethics, 14(4), 535-545

Parr, H. (2000) Interpreting the 'hidden social geographies' of mental health: ethnographies of inclusion and exclusion in semi-institutional places. Health \& Place, 6(3), 225-237.

Parr, H. (2011) Mental health and social space: Towards inclusionary geographies? Oxford: John Wiley \& Sons.

Perkins, E., Prosser, H., Riley, D. and Whittington, R. (2012) Physical restraint in a therapeutic setting; a necessary evil? International journal of Law and Psychiatry, 35(1), 4349.

Prior, L. (1988) The Architecture of the Hospital: A Study of Spatial Organization and Medical Knowledge, The British Journal of Sociology. 39, 1, 86-113.

Putkonen, A., Kuivalainen, S., Louheranta, O., Repo-Tiihonen, E., Ryynänen, O.P., Kautiainen, H. and Tiihonen, J. (2013) Cluster-randomized controlled trial of reducing seclusion and restraint in secured care of men with schizophrenia. Psychiatric Services, 64(9), 850-855. 
Quirk, A. and Lelliott, P. (2001) What do we know about life on acute psychiatric wards in the UK? A review of the research evidence. Social Science and Medicine, 53(12), 1565-1574.

Randall, D. \& McKeown, M. (2014) Failure to care: nursing in a state of liquid modernity? Journal of Clinical Nursing, 23, 766-767.

Rogers, A. and Pilgrim, D. (2014) A sociology of mental health and illness. $5^{\text {th }}$ Edition. Maidenhead: Open University Press/McGraw-Hill Education.

Rose, D., Evans, J., Laker, C., \& Wykes, T. (2015). Life in acute mental health settings: Experiences and perceptions of service users and nurses. Epidemiology and Psychiatric Sciences, 24(1), 90-96.

Rose, D., Perry, E., Rae, S., \& Good, N. (2017). Service user perspectives on coercion and restraint in mental health. BJPsych international, 14(3), 59-61.

Rose, N. (1996) Psychiatry as a political science: advanced liberalism and the administration of risk. History of the Human Sciences, 9(2), 1-23.

Russo, J. and Beresford, P. (2015) Between exclusion and colonisation: Seeking a place for mad people's knowledge in academia. Disability and Society, 30(1), 153-157.

Spandler, H. (2009) Spaces of psychiatric contention: a case study of a therapeutic community. Health \& Place, 15(3), 672-678.

Spradley, J. P. (1980) Participant Observation. New York: Holt, Rinehart and Winston.

Sweeney, A., Filson, B., Kennedy, A., Collinson, L. and Gillard, S. (2018) A paradigm shift: relationships in trauma-informed mental health services. Bjpsych Advances, 24(5), 319-333.

Szmukler, G. (2015) Compulsion and "coercion" in mental health care. World Psychiatry, 14(3), 259-261.

Tyler, T.R. (2006) Why people obey the law. Princeton, NJ: Princeton University Press. 
Vindrola-Padros, C. and Vindrola-Padros, B. (2018) Quick and dirty? A systematic review of the use of rapid ethnographies in healthcare organisation and delivery. BMJ Qual Saf, 27(4), 321-330.

Watson, S., Thorburn, K., Everett, M. and Fisher, K.R. (2014) Care without coercion-mental health rights, personal recovery and trauma-informed care. Australian Journal of Social Issues, 49(4), 529-549.

Weber, M. (1905/1958) The Protestant ethic and the spirit of capitalism. Trns. T. Parsons (1930). New York: Charles Scribner's Sons.

Wolch J. and Philo C. (2000), From distributions of deviance to definitions of difference; past and future mental health geographies. Health and Place, 6(4): 137-57. 